\title{
PROBLEMAS DE PLAUSIBILIDAD Y EVIDENCIA EMPÍRICA EN LOS RECIENTES ESTUDIOS CIENTÍFICOS SOBRE LA RELIGIÓN
}

\author{
LLUIS OVIEDO TORRÓ, OFM \\ Pontificia Universidad Antonianum, Roma
}

\begin{abstract}
RESUMEN. Las Ciencias Cognitivas de la Religión (RSE) han desarrollado desde hace casi veinte años explicaciones naturalistas sobre la mente y el comportamiento religioso, abriendo ese campo al análisis científico. Los estudiosos acostumbrados a comprender la religión desde una tradición más humanista o un enfoque hermenéutico, como teólogos, filósofos de la religión, y fenomenólogos pueden sentirse sorprendidos por la aplicación de métodos biológicos-evolutivos, cognitivos y neurológicos para explicar mejor la religión; a menudo perciben como extraño el uso de nuevos términos, conceptos y formas de entender la religión, más allá de los marcos tradicionales. El tiempo ha madurado para una evaluación de la plausibilidad que exhiben estas nuevas teorías, teniendo en cuenta sus respectivos marcos y la evidencia empírica reportada.
\end{abstract}

PALABRAS CLAVE: estudio científico de la religión, psicología cognitiva, antropología, evolución, reduccionismo.

\section{Issues concerning plaussiblity and empirical evidence in recent scientific study of religion-}

\begin{abstract}
Cognitive Science of Religion (CSR) has developed for almost twenty years naturalistic explanations on religious mind and behavior, opening that field to scientific scrutiny. Scholars used to study religion from a more humanistic tradition or a hermeneutic approach could feel surprised by the application of biological-evolutionary, cognitive and neurological means to better explain religion. Not too confident with the new approach, many traditional students of religion, like theologians, religion philosophers, phenomenologists, and even psychologists, were often dazzled by the exhibition of new terms, concepts and ways to understand religion, beyond the traditional frames. Time is ripe for an assessment on the plausibility that these new theories exhibit, taking into account their respective frameworks and the reported empirical evidence.
\end{abstract}

KEYWORDS: scientific study of religion, cognitive psychology, anthropology, evolution, reductionism.

La religión es una de las cuestiones más difíciles, tanto para la filosofía como para las ciencias. Lo mismo puede decirse respecto de la conciencia, o incluso, sencillamente, de la naturaleza humana. Desde la Ilustración los filósofos han tratado de dar una explicación «racional» de una realidad que — como sugieren algunas voces - ellos mismos habrían inventado. Tal intento continuará durante los siglos XIX y XX, dejando descontentos a la mayoría de los pensadores sobre las teorías 
resultantes de dichas exploraciones. Algo similar podría decirse con respecto de la naturaleza humana. Su «invención» es bastante reciente, como sostenía Michael Foucault, es decir se trata de un tópico que surge en el ambiente moderno. Una vez más la Ilustración intentó proporcionar teorías y explicaciones destinadas a comprender mejor esa "particularidad» del mundo natural, y los resultados han sido siempre incapaces de dar cuenta de forma «completa» y satisfactoria de la condición humana.

Los paralelismos entre los intentos de comprender mejor la religión y la naturaleza humana son evidentes en el periodo moderno. Respecto de la religión, han resultado siempre aporéticos y frustrantes: todavía se percibe una insatisfacción general ante las muchas propuestas disponibles. Por otro lado, los intentos de explicar la naturaleza humana dan lugar a otras dificultades: no se puede explicar el comportamiento humano sin tener en cuenta los contenidos conscientes; y sin pensamientos conscientes no se puede explicar el mundo físico y corporal; una dimensión llama a la otra, y la circularidad es inevitable. Sin embargo, sabemos de muchos intentos para obviar una de las dimensiones; una vez la víctima a sacrificar era el lado «externo», es decir, el cuerpo, la alteridad. Es lo que ocurrió en la mayor parte de la filosofía moderna Trascendental e Idealista, una tendencia que encuentra versiones actualizadas en varios programas de la filosofía del siglo XX. Pero, de repente, el estado de ánimo cambió, y el «partido fisicalista» se impuso, sacrificando los contenidos mentales y todo lo que podía estar relacionado con los procesos conscientes. Una vez más, el sujeto humano se vuelve demasiado intrincado y complejo para permitir una teoría de fácil solución ${ }^{1}$.

Sin embargo, los mencionados fracasos no deberían desalentar nuevos intentos en el estudio de la religión y la antropología, y de establecer relaciones entre ambos. En cualquier caso, la reflexión y la investigación en curso desde el inicio de la modernidad han ayudado en gran medida a conocer — más que a «explicar»— la religión y el ser humano, o al menos a proporcionar información sobre algunos de sus aspectos más intrigantes. No obstante, se debe deducir de todo ello que los esfuerzos modernos en la explicación de la religión y el ser humano hayan sido vanos y fútiles; más bien hay que reconocer que todos estos esfuerzos nos han ayudado a poner de relieve aspectos antes ignorados, asumiendo importantes distinciones; lo que nos ha hecho más conscientes de nuestros límites cognitivos ante tales complejidades. Una conciencia similar ha sido expresada recientemente, por ejemplo, en un trabajo colectivo en relación con el lenguaje humano y la falta de explicaciones convincentes sobre sus orígenes ${ }^{2}$. Algo análogo sucede en relación con la conciencia en las últimas dos décadas; quizás una de las voces más respetadas que se unen a la lista de escépticos acerca de las explicaciones disponibles en dicho campo es la de Thomas Nagel ${ }^{3}$.

1 Deacon, T. W., The Symbolic Species: The Co-evolution of Language and the Brain, Norton \& Co., New York, London 1997

2 Hauser, M. D., Yang, Ch., Berwick, R. C., Tattersall, I., Ryan, M. J., Watumull, J., Chomsky, N., and Lewontin, R. C., «The mystery of language evolution» en: Frontiers in Psychology, 2014, doi: 10.3389/ fpsyg.2014.00401.

3 Nagel, T., Mind and Cosmos: Why the materialist neo-Darwinian conception of nature is almost certainly false, Oxford University Press, Oxford New York 2012. 
El hecho es que los últimos veinte años han sido testigos de muchos intentos de «explicar la religión ${ }^{4}$, aunque tales esfuerzos, vistos desde cierta distancia, no proporcionan la evidencia esperada y el poder explicativo que prometían sus títulos. Sin embargo, la lista de propuestas se ha ampliado con creces. He preparado una nueva «revisión sistemática» —en prensa-que recoge más de 60 teorías naturalistas originales y con matices diferentes publicadas en los últimos 15 años tratando de «explicar la religión». Algo ha fallado en todo este programa, posiblemente una descripción más precisa de la naturaleza y la mente humana en las que se enmarca dicho estudio. Es preocupante que esta línea de investigación, a pesar de sus muchos defectos, haya sido utilizada de forma explícita con el fin de descalificar las creencias religiosas e incluso de justificar un programa de ateismo militante ${ }^{5}$.

Teniendo en cuenta este panorama introductorio, el presente ensayo intenta mostrar los límites que plantea el actual programa de estudio científico de la religión. En ese sentido, el método es eminentemente crítico, y tiene como objetivo mostrar que los varios defectos que pueden percibirse en el programa actual del nuevo estudio científico de la religión —básicamente en su versión cognitiva y evolucionista- se deben principalmente a una antropología deficitaria que, consciente o inconscientemente, ha servido de base para construir ese modelo. El principal problema es la metodología altamente reductiva aplicada hasta ahora, como resultado de un uso deficiente del principio de parsimonia, es decir la necesidad de limitar al máximo las explicaciones de los fenómenos bajo estudio.

\section{Cuestiones GENERALES EN TORNO AL ESTUdio COGNITIVO Y EVOLUCIONISTA DE LA RELIGIÓN}

El estudio en clave cognitiva y evolucionista de la religión representa un programa que ha madurado en casi veinte años y ha producido un importante corpus bibliográfico, con revistas y series de libros especializados. No es el objetivo del presente ensayo hacer una presentación de dichas claves y de las líneas principales de desarrollo de dicho programa; ya existen excelentes síntesis ${ }^{6}$. A grandes rasgos, hay que tener en cuenta que dicho programa se inscribe en la línea de una completa «naturalización» del hecho religioso, es decir, trata de explicarlo sin ninguna referencia trascendente o sin tener en cuenta el valor de las creencias que animan a las personas religiosas. A diferencia de otros intentos de describir el fenómeno religioso desde una óptica científica, la nueva orientación se sirve de un análisis de los esquemas o mecanismos mentales, y de los comportamientos que se asocian a la actitud religiosa, para tratar de explicarla a partir de dichos esquemas, que de

4 HINDE, R., Why gods persist: a scientific approach to religion, Routledge, London 1999; BoYeR, P., Religion Explained: The Evolutionary Origins of Religious Thought, Basic Books, New York 2001.

5 Dennetr, D., Breaking the Spell: Religion as a Natural Phenomenon, Viking, New York 2006; Shults, F., Theology after the Birth of God: Atheist Conceptions in Cognition and Culture, PalgraveMacmillan, London, New York 2014.

6 Watts, F. and Turner, L. (eds.), Evolution, Religion and Cognitive Science: Critical and Constructive Essays, Oxford University Press, Oxford, New York 2014; JonEs, J. W., Can Science Explain Religion? The Cognitive Science Debate, Oxford University Press Oxford, New York 2015. 
alguna manera canalizan las creencias trascendentes; o bien a partir de la norma evolutiva, que platea el significado de una conducta cuando sirve para mejorar las condiciones de supervivencia o de reproducción.

Las pautas de las que se sirve la nueva ciencia cognitiva y evolucionista de la religión seguramente proporcionan explicaciones de gran valor heurístico de algunos de los aspectos de la mente y del comportamiento religiosos, aunque no pueden pretender construir teorías generales y completas de todas las dimensiones implicadas en la experiencia religiosa. Seguramente las creencias religiosas se sirven de circuitos neuronales en la base de procesos cognitivos amplios, aunque se apliquen a campos específicos, como el conocimiento de factores trascendentes, lo que puede implicar una «re-utilización» de esquemas mentales cuyo origen y ámbito aplicativo original era mucho más simple o servía funciones más elementales. Algo parecido ha sido observado en otras aplicaciones cognitivas, como la capacidad de escribir o de leer, que implican una «re-utilización» de circuitos no previstos para dicho uso ${ }^{7}$.

También la atención a las valencias adaptativas de los comportamientos religiosos muestra claves de interés, incluso desde un punto de vista teológico. Seguramente dichas explicaciones se vinculan a la tradición del pensamiento funcionalista, que ya identificó, al menos desde Emile Durkheim funciones asociadas a la fe religiosa, tanto a nivel individual como social. En esta nueva fase, la orientación más biológica trata de identificar los elementos que podrían servir a una mejor adaptación de individuos o de grupos, o bien que pudieran conferir alguna ventaja a sus portadores a nivel de mejorar la capacidad reproductiva, y la mejor adaptación de la prole. Son muchos los puntos sugeridos a dicho respecto, como la capacidad de incentivar el comportamiento prosocial; la identificación de individuos más solidarios, y —al mismo tiempo- la corrección de aquellos más aprovechados; o bien las «virtudes» que se asocian a un buen parentesco.

De todos modos cierta insatisfacción queda en los estudiosos tradicionales de la religión —-teólogos, filósofos y fenomenólogos- ante dichas propuestas. Hay mucho que es simplemente ignorado y que sin duda alguna también contribuye a la experiencia religiosa, dimensiones que son sistemáticamente descuidadas y que siempre han tenido un papel relevante, como la capacidad simbólica, el marco cultural, la provisión de sentido y de esperanza, la capacidad de «afrontamiento" y muchas otras. Dicha parcialidad es preocupante, pero puede ser explicada a partir de la tendencia estándar en las ciencias a asumir un programa claramente reductivo, es decir, a reducir al mínimo los factores implicados a la hora de facilitar una explicación convincente y "económica», en el sentido de no dilapidar argumentos.

De todos modos, conviene ir más a fondo en nuestro intento de comprender los límites del nuevo estudio científico de la religión, en parte para neutralizar sus efectos más negativos para los creyentes, y en parte para corregir y ayudar a progresar en el estudio de la fe religiosa, como algo que también puede contribuir al avance teológico.

El proceso de recepción de esa línea de investigación ha alcanzado un nivel suficiente de madurez que permite evaluar sus propuestas y resultados. Varios intentos

7 Colagè, I., «The Human Being Shaping and Transcending Itself: Written Language, Brain, and Culture» en: Zygon, 50-4, 2015, pp. 1002-1021. 
ya han sido avanzados en vistas a una valoración crítica ${ }^{8}$. El debate en curso ofrece la oportunidad de discernir mejor los méritos y defectos en el material publicado, y como resultado, para profundizar en el diálogo interdisciplinar con las formas tradicionales en el estudio de la religión. Dos líneas críticas emergen es este panorama. La primera se refiere a los problemas que plantean las bases teóricas en las que se apoyan los desarrollos del nuevo programa de estudio científico de la religión, y que básicamente se refiere al marco cognitivo y al antropológico. La segunda analiza los niveles de evidencia que han facilitado hasta la fecha dichos estudios para apoyar sus teorías. El presente ensayo se concentra en la primera línea, y pantea brevemente la revisión de los datos empíricos que confieren plausibilidad científica a dichos estudios.

\section{CUESTIONES COGNITIVAS Y ANTROPOLÓGICAS}

La primera estrategia crítica se concentra en la matriz teórica, o los marcos en que se inscriben los estudios cognitivos y biológicos de la religión. Dos casos merecen un escrutinio más detallado: los modelos cognitivos aplicados en la primera generación de estos estudios; y los modelos antropológicos que han guiado más la construcción de teorías en ese campo. El punto es claro: si los marcos teóricos que sirvieron de base para los nuevos desarrollos en el estudio de la religión están sujetos a fuertes críticas, entonces las teorías derivadas van a sufrir seriamente en su capacidad explicativa. Esto es evidente en lo que se refiere a las teorías conexionistas de la mente y su estructura neurológica, un modelo ampliamente superado desde otros estudios de la mente en años recientes. Por otro lado, la antropología científica ha desarrollado en los últimos diez años modelos de niveles múltiples que ofrecen puntos de vista más plausibles sobre la naturaleza humana y su compleji$\operatorname{dad}^{9}$. A partir de estos desarrollos las anteriores propuestas más reductivas, al igual que las deudoras de la psicología evolutiva, la genética o la neurología, aparecen como poco satisfactorias, cuando no fuera de contexto, cuando se desarrolla un

8 LaIdLAW, J., «A well-disposed social anthropologist problem with the "cognitive science of religion" ", en: Whitehouse, H., and Laidlaw, J., Religion, Anthropology, and Cognitive Science, Caroline Academic Press, Durham, NC 2007, 211-246; DAY, M., «Let's Be Realistic: Evolutionary Complexity, Epistemic Probabilism, and the Cognitive Science of Religion» en: Harvard Theological Review, 100-1, 2007, pp. 47-64; BARRETT, N. F., «Toward an Alternative Evolutionary Theory of religion: Looking Past Computational Evolutionary Psychology to a Wider Field of Possibilities» en: Journal of the American Academy of Religion, 78-3, 2010, pp. 583-621; VISALA, A., Naturalism, Theism and the Cognitive Study of Religion: Religion Explained? Ashgate, Farnham UK, Burlington VT, 2011; VAN StYKE, J.A., The Cognitive Science of Religion, Ashgate, Farnham UK, Burlington VT, 2011; SchÜLER, S., Religion, Kognition, Evolution: Eine religionswissenschaftliche Auseinandersetzung mit der Cognitive Science of Religion, Kohlhammer, Stuttgart, 2012; TuRK, M., Being Religious: Cognitive and Evolutionary Theories in Historical Perspective, Pickwick, Eugen, OR, 2013; WatTs, F. and TuRner, L. (eds.), Evolution, Religion and Cognitive Science: Critical and Constructive Essays, Oxford University Press, Oxford, New York, 2014; Sмrтн, A.C.T., Thinking About Religion: Extending the Cognitive Science of Religion, Palgrave-Macmillan, London, New York 2014; JonEs, J.W., Can Science Explain Religion? The Cognitive Science Debate, Oxford University Press Oxford, New York, 2015.

9 JablonKa, E. and Lamb, M., Evolution in four dimensions: Genetic, epigenetic, behavioral, and symbolic variation in the history of life, MIT Press, Cambridge, MA, 2005. 
modelo más integral, donde cada dimensión puede encontrar su propio lugar y función.

La evolución descrita significa mucho para la actual ciencia cognitiva y evolucionista de la religión: una nueva comprensión de la cognición y de la complejidad de la evolución humana exige una revisión profunda de los modelos que se construyeron sobre los conocimientos científicos de finales de los noventa o principios del nuevo milenio.

\subsection{Cuestiones relativas al marco cognitivo}

Los avances en las ciencias cognitivas sirvieron de base para aplicaciones en la ciencia cognitiva de la religión. Desde la publicación del libro de Jerry Fodor sobre la modularidad de la mente ${ }^{10}$, hasta su propia refutación años más tarde ${ }^{11}$, podría describirse un arco que cubre los cambiantes estados de ánimo dominantes en ese campo. El modelo original que se desarrolló en las primeras etapas de la psicología cognitiva ha proporcionado un buen marco para los estudiosos que trataban de describir la religión en términos cognitivos; esto es bastante claro en el caso de Pascal Boyer y varios otros. Sin embargo, el modelo modular, la propuesta conexionista, e incluso el uso de modelos computacionales para describir cómo funciona la mente humana, han sido objeto de fuertes críticas en los últimos años ${ }^{12}$.

Algunas tendencias actuales en la ciencia cognitiva describen la mente desde un programa «multi-componente» que incluye un conjunto de dimensiones que deben tenerse en cuenta más allá de posiciones demasiado reductivas. Estos desarrollos tienen un profundo impacto en cualquier modelo que intente explicar la naturaleza y el funcionamiento de la religión, y dado que la producción disponible se basa casi exclusivamente en los primeros desarrollos de la biología evolutiva y de la ciencia cognitiva, las propuestas existentes sufren una urgente necesidad de revisión y actualización.

Una segunda característica a tener en cuenta se refiere a la reducción del pensamiento religioso a sus niveles más intuitivos e inconscientes. Una impresión general, después de tratar durante muchos años con el nuevo estudio científico de la religión, es que el énfasis recae en las dinámicas inconscientes, las ideas intuitivas, y en rasgos de comportamiento casi mecánicos. Todo lo que es consciente o vinculado a la dimensión reflexiva a menudo se descuida o se vuelve secundario en ese análisis de la mente religiosa. Por otra parte, la libertad de elegir y la toma de decisiones pierden significado en ese nuevo marco. No es de extrañar que los aspectos claramente relacionados con la dimensión consciente pasen a un segundo plano, como algo extraño a la mirada científica. Tal descuido es bastante sorprendente, ya que, en muchos casos, los estudiosos de la ciencia cognitiva de la religión han citado los llamados «esquemas cognitivos duales» elaborados por la psicología

10 Fodor, J., Modularity of Mind: An Essay on Faculty Psychology, MIT Press, Cambridge, MA, 1983.

1 Fodor, J., The mind doesn't work that way, MIT Press, Cambridge, MA, 2000.

12 Palm, G., «Neural Information Processing in Cognition: We Start to Understand the Orchestra, but Where is the Conductor?» en: Frontiers in Computational Nuerosciecne, 2016, doi: 10.3389/ fncom.2016.00003 
experimental desde los años setenta, y que hacen hincapié en una doble dimensión en el funcionamiento de la cognición humana: rápida y lenta; intuitiva y reflexiva; espontánea y calculada. Sin embargo, parecía que sólo la dimensión intuitiva o inconsciente funciona en la mente religiosa, y la otra dimensión queda de alguna manera profundamente condicionada por aquella. Tal descripción se aleja claramente de cómo las personas religiosas elaboran sus creencias y toman decisiones pertinentes, que a menudo tienen una clara recaída moral ${ }^{13}$.

La sospecha es que la ciencia puede introducir alguna distorsión en el estudio de la religión, al igual que sucede en otras áreas de investigación. Como consecuencia, sería aconsejable mantener una cierta cautela al acceder a esas nuevas perspectivas $^{14}$. La gran pregunta es, entonces, si el punto de vista científico no puede evitar una reducción unilateral de ciertos rasgos humanos, que deben ser ignorados como condición para llegar a un análisis más preciso. En otras palabras, la cuestión que se plantea ahora es si el enfoque científico no puede hacer otra cosa, y por lo tanto tenemos que asumir esta limitación como un precio a pagar por la exactitud y el carácter comprobable de sus puntos de vista y de los resultados que presenta.

\subsection{La cuestión antropológica en sus desarrollos más recientes}

Parte del problema con las propuestas estándar en el paradigma cognitivo y bioevolucionista actual es el marco antropológico que presuponen, que a menudo se vuelve demasiado simplista, ignorando rasgos que casi todo el mundo da por sentado. Este punto necesita una aclaración. El debate antropológico ha procedido en los últimos veinte años como un intento de discernir el mejor acceso a la naturaleza humana, y si conviene deshacerse de algunos rasgos humanos que siempre se han considerado importantes en la tradición occidental. De hecho, el enfoque científico no parece capaz de reconocerlos o no está dispuesto a considerarlos. La cuestión es si podemos obtener una mejor comprensión de la naturaleza humana evitando los escollos del reduccionismo excesivo, pero manteniendo al mismo tiempo un buen nivel científico.

Muchos títulos se han publicado en los últimos años para denunciar una aplicación incorrecta de los modelos científicos que tratan de explicar la naturaleza humana. Raymond Tallis ha sido uno de los más críticos en esta campaña contra el uso incorrecto de teorías biológicas o neurológicas ${ }^{15}$. No es el único en asumir un tono tan crítico. Después de una larga lista de pensadores «humanistas» que han participado en tales esfuerzos desde principios de los noventa, cabe citar algunas incorporaciones recientes, como: Marilynne Robinson, Absence of Mind; Paolo

13 Oviedo, Ll., «Religious Cognition as a Dual-Process: Developing the Model» en: Method and Theory in the Study of Religion, 27-1, 2015, pp. 31-58; VAINIO, O-P., "What Does Theology Have to Do With Religion? Dual-process Accounts, Cognitive Science of Religion and a Curious Blind Spot in Contemporary Theorizing» en: Open Theology, 2, 2016, pp. 106-112.

14 Bolger, R., Kneeling at the Altar of Science: The Mistaken Path of Contemporary Religious Scientism, Wipf and Stock, Eugene, OR, 2012.

15 Tallis, R., Aping Mankind: Neuromania, Darwinitis and the Misrepresentation of Humanity, Acumen, Durham, 2011. 
Legrenzi y otros, Neuromania: On the Limits of Brain Science ${ }^{16}$; Estos y otros títulos constituyen en sí mismos una protesta en contra de una tendencia incapaz de describir la verdadera realidad humana, haciendo caso omiso de sus rasgos más característicos, como su pensamiento consciente, su lenguaje y capacidad de comunicación, y la generación de mundos culturales en los que está incrustada.

Tallis ofrece algunas ideas respecto a una antropología alternativa que podría satisfacer las condiciones mencionadas: la auto-conciencia aparece en el centro, junto con la estructura social de los seres humanos, con toda su riqueza y complejidad. En cualquier caso, no es difícil encontrar propuestas alternativas que tratan de construir antropologías más completas, evitando las trampas del reduccionismo excesivo o haciendo caso omiso de las características centrales de la vida humana. Desarrollos recientes apuntan más bien a modelos «multinivel» capaces de dar cuenta de distintos aspectos de la compleja realidad humana. Varios investigadores han seguido las propuestas de Eva Jablonka y Marion Lamb, Evolución en cuatro dimensiones: Genética, epigenética, del comportamiento, y la variación simbólica en la historia de la vida ${ }^{17}$. Como plantea dicho título, la evolución humana ha estado marcada por factores biológicos y culturales, en referencia al mundo de los símbolos.

El principal reto de la antropología contemporánea consiste en integrar el enfoque de lo humano en una amplia red de varias dimensiones, que han influido en su historia evolutiva, y que apenas se han tenido en cuenta en los intentos científicos anteriores. La lista debe incluir, al menos: los procesos conscientes, el lenguaje, la provisión de sentido, y el contexto cultural. Una abundante literatura apunta en los últimos años en esa dirección, como por ejemplo los estudios sobre la capacidad simbólica; estudios que subrayan la co-evolución genética y cultural, o consideran la dimensión cultural como un elemento central en la comprensión de lo humano; los intentos de tratar el lenguaje como un rasgo humano específico; y los estudios sobre el sentido y la toma de decisiones. Es significativo cómo un debate reciente entre los biólogos evolutivos muestra cómo la cuestión antropológica puede ayudar a reconfigurar el paisaje evolutivo. El punto es que, cuando los seres humanos son parte del tema, entonces no es el proceso estándar de la evolución el que ayuda a comprender la naturaleza humana, sino que es el estudio de los seres humanos el que ayuda a comprender mejor y ampliar un enfoque demasiado estrecho del proceso adaptativo ${ }^{18}$.

Toda esta investigación apuesta claramente por un cambio de marco en el estudio científico de la religión, una vez se han integrado estos nuevos desarrollos. La idea central es que una antropología más «humana» obliga a un enfoque distinto de la religión.

16 Robinson, M., Absence of Mind: The Dispelling of Inwardness from the Modern Myth of the Self, Yale University Press, New Haven, London, 2010; Legrenzi, P., Umiltà. C. and Anderson, F., Neuromania: On the Limits of Brain Science, Oxford University Press, Oxford, New York, 2011.

17 Jablonka, E. and Lamb, M., Evolution in four dimensions: Genetic, epigenetic, behavioral, and symbolic variation in the history of life, MIT Press, Cambridge, MA, 2005.

18 Laland, K., Wray, G. A., et al. "Does evolutionary theory need a rethink?» en: Nature 514, 2014, pp. 161-164. 
En este breve ensayo hay escasas posibilidades de revisar la considerable cantidad de literatura que apunta a la extensión de los modelos más reductivos de lo humano, su evolución y los factores que intervienen en su comportamiento real. Sin duda una mejor antropología podría proporcionar un fundamento más exacto para el estudio cognitivo de la religión.

Después de este breve recorrido por la antropología contemporánea, una conclusión es que todo estudio acerca de un rasgo o comportamiento humano debe asumir de manera consciente qué marco es más apropiado para el propio programa de investigación. A este respecto, el estudio científico de la religión, al igual que el de la moral o del amor, debe explicitar el marco antropológico dentro del cual se mueve: si se trata del biológico, neurológico — ambos bastante reductivos - o uno más integral y complejo. Esta es una opción muy arriesgada, y un dilema difícil. La mayor parte del estudio cognitivo y bio-evolucionista de la religión ha sguido una pauta reductiva, y esto es evidente cuando se analizan los principales estudios publicados; en general han tratado de «explicar la religión» a costa de perder de vista la verdadera naturaleza de los seres humanos. Aunque dicho ejercicio es legítimo, debe admitir que se trata de un enfoque basado en lo que podría ser diseñado como un método ceteris paribus, o de «dejar todas las demás variables constantes». Si se pueden aislar algunos mecanismos cognitivos o reglas biológicas de todo el resto de los factores humanos complejos y las interacciones sociales, entonces se puede obtener alguna explicación acerca de cómo la mente y el comportamiento religioso procede dentro de esos estrechos márgenes. El problema es que el investigador tiene que dar más pasos para integrar otros factores que intervienen en el proceso, o simplemente ignorarlos y desestimar su importancia o efecto real; sin embargo, este paso significa una elección consciente de una antropología reductiva, o una visión conscientemente limitada de la complejidad humana.

El enfoque descrito podría ser comparado con algunos juegos imaginarios, como por ejemplo el llamado «juego de la tierra plana» (flatland), o un intento de visualizar la realidad cuando se reduce a sólo dos dimensiones. Esto puede ser de gran ayuda para comprender mejor la naturaleza de una realidad más compleja, pero sólo cuando sus practicantes están convencidos de que el suyo es sólo un ejercicio que ayuda a comprender mejor la complejidad de los procesos reales, en los que están implicadas más dimensiones.

\section{LA CUESTIÓN DE LA EVIDENCIA EMPÍRICA}

Un segundo motivo de preocupación se refiere a los niveles de evidencia reunidos por las nuevas propuestas de dicha ciencia. Este punto afecta a la credibilidad o poder explicativo pretendido por esas teorías. Incluso si una parte de la construcción teórica actual puede obviar la evidencia empírica o experimental, hay algunos puntos que podrían ser verificados o pueden proporcionar predicciones que serían objeto de evaluación. En este caso, dos principios centrales en el llamado «modelo estándar» merecen un escrutinio crítico: el primero se refiere a la idea de que las religiones evolucionaron debido a su capacidad para incentivar un comportamiento pro-social; y el segundo vincula las ideas religiosas a la «teoría de la mente». En el 
primer caso, la evidencia disponible es bastante concluyente y la discusión actual no logra dilucidar la cuestión. Una reciente revisión sistemática extensa de estudios publicados ha demostrado que la religión se vuelve pro-social sólo en algunos casos y no para todo el mundo ${ }^{19}$ (Oviedo 2016). La misma revisión del archivo histórico muestra la presencia de muchas religiones sin rasgos prosociales.

En el segundo caso, es decir, en qué medida la religión está relacionada con la "teoría de la mente», carecemos por ahora de pruebas que demuestren que las personas con deficiencias en la capacidad de reconocer los estados mentales de los otros, algo característico de los individuos en el trastorno del espectro autista (ASD), sean, en promedio, menos religiosas. Es probable que estas personas logren desarrollar una forma propia de cognición religiosa, o aprendan de forma diferente a ser religiosas, pero, en principio, no resultan «religiosamente desfavorecidas». Algunos datos recientes apuntan a correlaciones moderadas entre los niveles de empatía en una escala estandarizada e indicadores de religiosidad, pero en todo caso pueden ser entendidas en ambos sentidos: es decir que los sujetos más religiosos en algunas tradiciones que enfatizan la compasión, podrían ser más sensibles hacia aquellos en mayor dificultad. Sin embargo la experiencia y la observación nos confirman que en muchos casos la orientación religiosa se especializa, y que quien sigue una vía más mística, acaba por descuidar la de la caridad.

La crítica anterior no significa que las ciencias cognitivas de la religión no tienen nada que ofrecer a los estudiosos que se mueven en campos paralelos en el estudio religioso. El discernimiento propuesto debe tratar de distinguir las contribuciones útiles para comprender mejor la fe religiosa, que son bastantes, y aquellos que es mejor dejar de lado. El verdadero problema, desde mi punto de vista, es la postura reduccionista que a menudo detentan los nuevos estudiosos de la religión. Es una lástima, ya que sus contribuciones tienen un valor explicativo dentro del marco en el que se mueven, pero no anulan las explicaciones complementarias y puntos de vista hermenéuticos que se mueven en un escenario más amplio.

Pontificia Universidad Antonianum. Roma loviedo@antonianum.eu

Lluis Oviedo Torró, OFM

[Artículo aprobado para publicación en diciembre de 2016]

19 Oviedo, Ll., «Religious attitudes and prosocial behavior: A systematic review of published research» en: Religion, Brain \& Behavior, 6-2, 2016, pp. 169-184. 